Check for updates

Cite this: Chem. Sci., 2019, 10, 737

๑ All publication charges for this article have been paid for by the Royal Society of Chemistry

\section{Growth of ZIF-8 on molecularly ordered 2-methylimidazole/single-walled carbon nanotubes to form highly porous, electrically conductive composites $\uparrow$}

\author{
James E. Ellis, ${ }^{a}$ Zidao Zeng, ${ }^{a}$ Sean I. Hwang, ${ }^{a}$ Shaobo Li, (DD b Tian-Yi Luo, ${ }^{\text {b }}$ \\ Seth C. Burkert, ${ }^{a}$ David L. White, ${ }^{a}$ Nathaniel L. Rosi, (D) Jeremiah J. Gassensmith (D) bc \\ and Alexander Star (D)*a
}

\begin{abstract}
The combination of porosity and electrical conductivity in a single nanomaterial is important for a variety of applications. In this work, we demonstrate the growth of ZIF-8 on the surface of single-walled carbon nanotubes (SWCNTs). The growth mechanism was investigated and a molecularly ordered imidazole solvation layer was found to disperse SWCNTs and promote crystal growth on the sidewalls. The resultant ZIF-8/SWCNT composite demonstrates high microporosity and electrical conductivity. The ZIF-8/SWCNT composite displayed semiconducting electrical behavior and an increase in sensor sensitivity toward ethanol vapors versus pristine SWCNTs.
\end{abstract}

Received 7th September 2018

Accepted 24th October 2018

DOI: $10.1039 /$ c8sc03987a

rsc.li/chemical-science

\section{Introduction}

A material that combines the physical properties of periodic porosity and electrical conductivity is an appealing candidate for a variety of applications including sensors, photovoltaics, thermoelectrics, electrocatalysts, and electrical energy storage materials. Electrically conductive metal-organic frameworks (MOFs) contain both of these physical properties; ${ }^{1}$ however, the mechanism of electrical conductivity found in most conductive MOFs, described as charge hopping, results in a much lower charge mobility than band transport materials. Charge hopping limits MOF electrical conductivity to high electrical fields and short distances, thus limiting their field of application. A potential solution to the low charge mobility inherent to conductive MOFs is to hybridize MOFs with band transport materials like carbon nanotubes (CNTs), thus improving electrical transduction in MOF-based sensors and electrocatalysts.

In this study, a zeolitic imidazolate framework (ZIF-8) was chosen as the model MOF because of its water stability, ease of synthesis, and well-documented characterization. ZIF-8 is composed of $\mathrm{Zn}$ (II) ions and 2-methylimidazole (2mIM) linkers,

${ }^{a}$ Department of Chemistry, University of Pittsburgh, Pittsburgh, PA 15260, USA. E-mail: astar@pitt.edu

${ }^{b}$ Department of Chemistry and Biochemistry, University of Texas at Dallas, TX 75080, USA

'Department of Bioengineering, University of Texas at Dallas, TX 75080, USA $\dagger$ Electronic supplementary information (ESI) available: Additional TEM, PXRD, Raman spectroscopy, and zeta potential measurement of synthesized materials; comparison table of this work with other conductive MOFs. See DOI: $10.1039 / \mathrm{c} 8 \mathrm{sc} 03987 \mathrm{a}$ and has been studied as both a gas storage material as well as a biomimetic mineralization layer material. ${ }^{2-5}$ First reports of ZIF-8 employed a solvothermal synthesis in DMF or DEF; ${ }^{6,7}$ however, room temperature aqueous syntheses that yield highly-crystalline ZIF-8 have recently been investigated., ${ }^{8,9}$

Several examples of MOF/CNT hybrid composites have been synthesized. Early reports found that the inclusion of CNTs into large MOF crystals enhance gas adsorption compared to the pristine MOF species. ${ }^{\mathbf{1 0}, 11}$ Shim and coworkers were the first to demonstrate a core-shell composite material through homogeneous decoration of small ZIF-8 crystals on PVP-dispersed multi-walled carbon nanotubes (MWCNTs). ${ }^{12}$ MOF/MWCNT hybrids have been applied toward electrocatalysis and energy storage because they are electrically conductive, high surface area materials. ${ }^{13-16} \mathrm{~A}$ pyrolysis step is added to some MOF/ MWCNT composites in order to convert the MOF shell into catalytically active metal nanoparticles and heteroatom-doped carbon domains. ${ }^{17-19}$ A MIL-101/single-walled carbon nanotube (SWCNT) composite has been synthesized by adding SWCNTs to the MOF precursor components. ${ }^{20} \mathrm{An}_{2}$ storage improvement was observed for the composite. Unlike MWCNTs, which have metallic electrical characteristics, smaller diameter SWCNTs can have either a metallic or semiconducting electronic structure depending on their chirality ${ }^{21}$ For this reason, they are purified based on chirality and applied toward electrical devices such as sensors and field-effect transistors. ${ }^{22-27}$ SWCNTs have excellent electrical characteristics (charge mobility, charge carrier concentration, etc.) and consist almost entirely of surface atoms, thereby making their electronic structure extremely sensitive to the 
surrounding chemical environment. To our knowledge, only two MOF/single-walled carbon nanotube (SWCNT) composite has been synthesized $;^{\mathbf{2 0} 28}$ however, no investigation of MOF growth on the surface of SWCNTs has been reported. Gassensmith and coworkers investigated the growth of ZIF-8 on tobacco mosaic virus, ${ }^{29}$ a one-dimensional bionanoparticle, and discovered that careful control of the MOF precursor ligand/ metal ratio significantly affects the resulting morphology of the composite. ${ }^{30}$ For this reason we chose to composite ZIF-8 with SWCNTs to fabricate a porous, semiconducting nanowire that could serve as a promising material for chemical sensing.

\section{Results and discussion}

In this work, all reactions were performed in ambient and aqueous conditions. Oxidized SWCNTs (ox-SWCNTs) were used in order to obtain well-dispersed carbon nanotubes in aqueous solution. Ox-SWCNTs are approximately 3 atomic\% of oxygen in the form of hydroxyl and carboxyl groups on their sidewall and termini. Sonication of ox-SWCNTs in polar solvents promotes dispersive forces, allowing separation of large carbon nanotube agglomerates into smaller bundles and individual ox-SWCNTs. A typical ZIF-8 synthesis involves careful control of zinc salt and 2-methylimidazole (2mIM) concentrations and $\mathrm{Zn}: 2 \mathrm{mIM}$ molar ratio. The addition of ox-SWCNTs as a template adds a complicating factor to the synthesis, wherein the order in which the precursors are added plays an important synthetic role. When ox-SWCNTs are mixed with the zinc salt first or both precursors simultaneously, they immediately re-agglomerate and precipitate out of solution before any ZIF- 8 forms. This effect is caused by salt-mediated contraction of the hydration layer around the charged ox-SWCNTs. However, when oxSWCNTs are first mixed with 2mIM, they remain dispersed in solution for minutes after adding Zn salt (Fig. S1†). It has been previously observed through molecular dynamics simulations that 1-ethyl-3-methylimidazolium tetrafluoroborate $\left(\mathrm{EMI}^{+} \mathrm{BF}_{4}{ }^{-}\right)$ forms an external solvation layer around SWCNTs through $\pi-\pi$ interaction between the SWCNTs and $\mathrm{EMI}^{+} .{ }^{31}$ Raman spectroscopy demonstrated that 2mIM-saturated ox-SWCNTs undergo a $\mathrm{G}$ peak shape change and red-shift as compared to pristine oxSWCNTs (Fig. S2 $\dagger$ ). The ox-SWCNT spectrum has a single G peak which is indicative of SWCNT bundling; in contrast, the G peak of the 2mIM/ox-SWCNT material splits into $\mathrm{G}^{-}$and $\mathrm{G}^{+}$ peaks, which is a signature of single, unbundled SWCNTs. ${ }^{32,33} \mathrm{~A}$ redshift in carbon nanotube $G$ peak is an indication of $n$ doping, which may be the result of charge transfer between $2 \mathrm{mIM}$ and ox-SWCNTs. ${ }^{34}$ Zeta potential measurements indicate that $2 \mathrm{mIM}$ does not diminish the negative surface charge of SWCNTs (Table S1†).

A synthesis was performed with a large excess of ox-SWCNT to determine whether a similar solvation layer formed between 2mIM and SWCNTs could be coordinated and crystalized by zinc ion addition (Table 1, material 1). If a 2mIM solvation layer forms around ox-SWCNT through $\pi$-stacking, then an excess amount of ox-SWCNTs should nucleate all available $\mathrm{Zn}^{2+}$ ions within the solvation layer and prevent the formation of ZIF-8 crystals in solution. The ox-SWCNTs and 2mIM solutions were
Table 1 Experimental parameters of synthesized materials

\begin{tabular}{lllll}
\hline Material & $\begin{array}{l}\text { Zinc } \\
\text { source }\end{array}$ & $\begin{array}{l}{[\mathrm{Zn}]} \\
(\mathrm{mM})\end{array}$ & $\begin{array}{l}\mathrm{Zn}: 2 \mathrm{mIM} \text { mol. } \\
\text { ratio }\end{array}$ & $\begin{array}{l}\mathrm{Zn}: \text { SWCNT } \\
(\mathrm{mmol}: \mathrm{mg})\end{array}$ \\
\hline 1 & $\mathrm{Zn}\left(\mathrm{NO}_{3}\right)_{2}$ & 20 & $1: 32$ & $1: 7.5$ \\
2 & $\mathrm{Zn}(\mathrm{OAc})_{2}$ & 20 & $1: 40$ & $1: 1$ \\
3 & $\mathrm{Zn}\left(\mathrm{NO}_{3}\right)_{2}$ & 20 & $1: 40$ & $1: 1$ \\
4 & $\mathrm{Zn}\left(\mathrm{NO}_{3}\right)_{2}$ & 20 & $1: 8$ & $1: 1$ \\
5 & $\mathrm{Zn}(\mathrm{OAc})_{2}$ & 20 & $1: 8$ & $1: 1$ \\
6 & $\mathrm{Zn}(\mathrm{OAc})_{2}$ & 10 & $1: 40$ & $1: 1$ \\
7 & $\mathrm{Zn}(\mathrm{OAc})_{2}$ & 6.6 & $1: 40$ & $1: 3.3$ \\
\hline
\end{tabular}

first combined prior to adding the zinc nitrate hexahydrate solution. The reaction was left unstirred for 4 hours. The reaction suspension was centrifuged to remove the mother liquor, then washed with water and methanol. TEM of material 1 shows dispersed nanotubes with a thick $(\sim 10 \mathrm{~nm})$ diameter (Fig. S3 $\dagger$ ). Powder X-ray diffraction (PXRD) of this material showed a dominant single peak at $2 \theta=17.78^{\circ}(d$-spacing $=4.98 \AA)$ and very small peaks relating to ZIF-8 formation (Fig. S4 $\dagger$ ). Fukushima et al. have previously reported the molecular ordering of molten salts (in their case, imidazolium-based ionic liquids) by SWCNTs. ${ }^{35}$ They observed a single XRD peak with $d$ spacing of $4.60 \AA$, which they attributed to unimodal long-range molecular ordering of plane-to-plane separated imidazolium ions without polycrystalline character. A similar molecular ordering phenomenon occurs between SWCNTs, 2mIM, and $\mathrm{Zn}^{2+}$. According to XRD, the $d$-spacing of this material is $4.98 \AA$, which is large for $\pi$-stacked 2mIM. When ox-SWCNTs were mixed with the same amount of 2mIM, but zinc nitrate was not subsequently added, no XRD peak was detected. This observation, in addition to the larger than expected $d$-spacing, implies that the $\mathrm{Zn}^{2+}$ ions play a role in the long-range ordering of $2 \mathrm{mIM}$ around the ox-SWCNTs. Raman spectroscopy of the crystalline 2mIM/ox-SWCNT material 1 demonstrated the same G peak shape change and red-shift as the 2mIM-saturated SWCNTs compared to untreated ox-SWCNTs (Fig. S2 $\dagger$ ).

Scheme 1 illustrates the effect order of precursor addition has on ox-SWCNT. The agglomeration of ox-SWCNTs caused by addition of zinc salt before 2mIM affects the resulting morphology of the ZIF-8/SWCNT, such that large ZIF-8 crystals grow on islands of agglomerated ox-SWCNTs rather than along the nanotube axis (Fig. S5 $\dagger$ ). In all later syntheses described in this work, 2mIM-saturated ox-SWCNTs are used as the precursor material in order to prevent agglomeration, to promote nucleation of ZIF-8 crystals according to pathway \#2 (Scheme 1), and to prevent $2 \mathrm{mIM}$ from further $\pi$-stacking on oxSWCNTs, which would alter the $\mathrm{Zn}: 2 \mathrm{mIM}$ ratio.

Factors that are known to influence ZIF growth, size, and morphology in water-based synthesis include zinc precursor source, zinc concentration, zinc to $2 \mathrm{mIM}$ ratio, and temperature. ${ }^{9,36}$ In this work, zinc(II) acetate and zinc(II) nitrate hexahydrate were used as zinc sources. In order to synthesize a ZIF-8/ SWCNT composite, a 1:40 $\mathrm{Zn}: 2 \mathrm{mIM}$ ratio and total zinc concentration of $20 \mathrm{mM}$ was used for each zinc source (materials 2 and 3). The zinc to ox-SWCNT ratio used was $1 \mathrm{mmol}$ $\mathrm{Zn}$ : $1 \mathrm{mg}$ ox-SWCNT. The reactions were left unstirred at room 

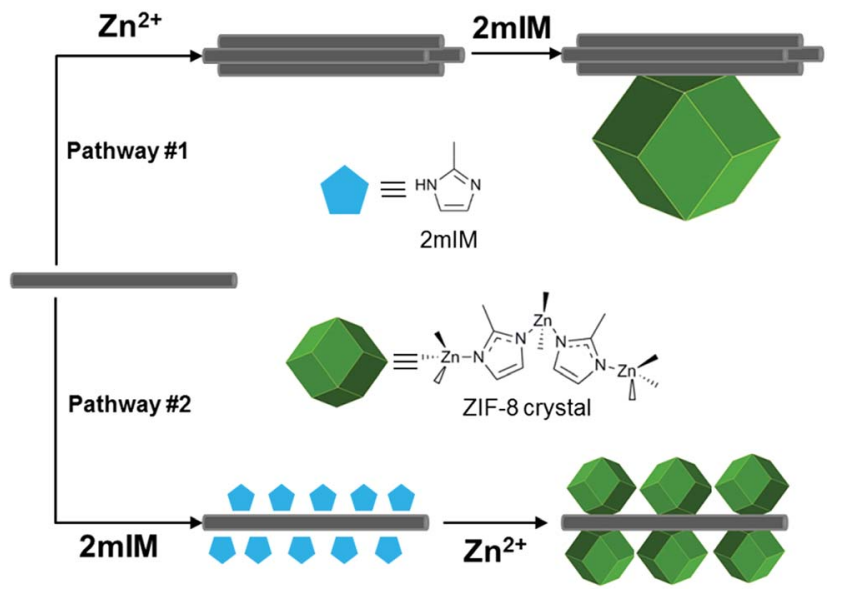

Scheme 1 The effect of precursor order. Pathway \#1 results in oxSWCNT agglomeration and precipitation, which results in a decreased ZIF-8/SWCNT interface. Pathway \#2 results in a $2 \mathrm{mIM}$ solvation layer around ox-SWCNTs, which promotes ZIF- 8 crystal growth on the sidewall. Not drawn to scale.

temperature for 24 hours. The reaction precipitates were collected with centrifugation and washed in water and methanol. The morphology of the resulting products of these syntheses can be described as large ZIF- 8 crystals $(1-1.5 \mu \mathrm{m})$ threaded with SWCNTs (Fig. 1 and S6†). TEM images show crystal growth occurs along a linear path, which is consistent with growth along a CNT axis. XRD of the ZIF-8/SWCNT composites made from both zinc sources contain characteristic ZIF-8 diffraction peaks (Fig. 2). The product composition of each synthesized material determined by PXRD is listed in Table S2. $\uparrow$ The peak widths of the ZIF-8/SWCNT composite made with zinc nitrate hexahydrate are $\sim 3$ times broader than the composite made with zinc acetate. The XRD peak broadening of ZIF-8/SWCNT synthesized from zinc nitrate can be attributed to the smaller crystal domain size of the composite. TEM and SEM of the zinc nitrate ZIF-8/SWCNT composite

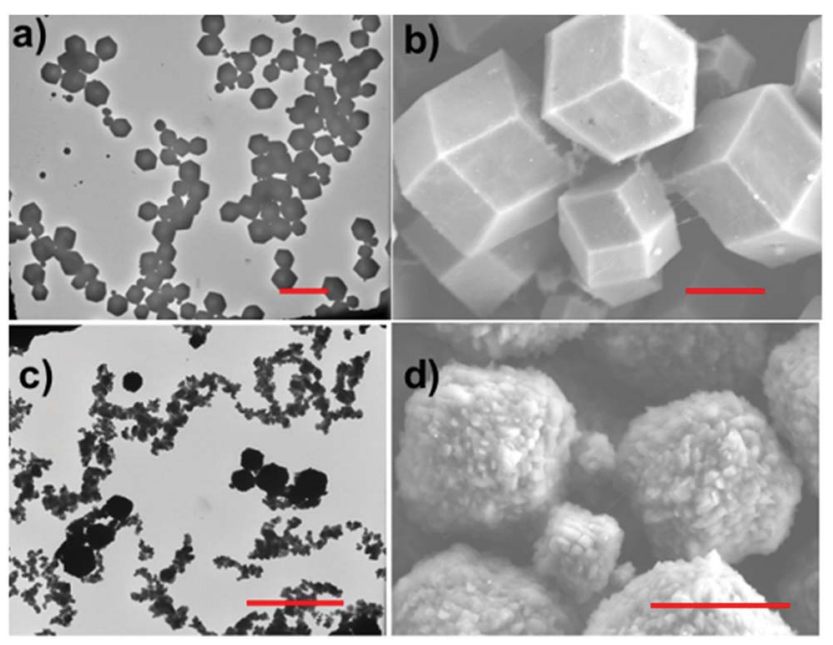

Fig. 1 TEM and SEM of ZIF-8/SWCNT. ( $a$ and b) Material 2 and (c and d) material 3. Scale bars are (a and c) $5 \mu \mathrm{m}$ and (b and d) $1 \mu \mathrm{m}$.

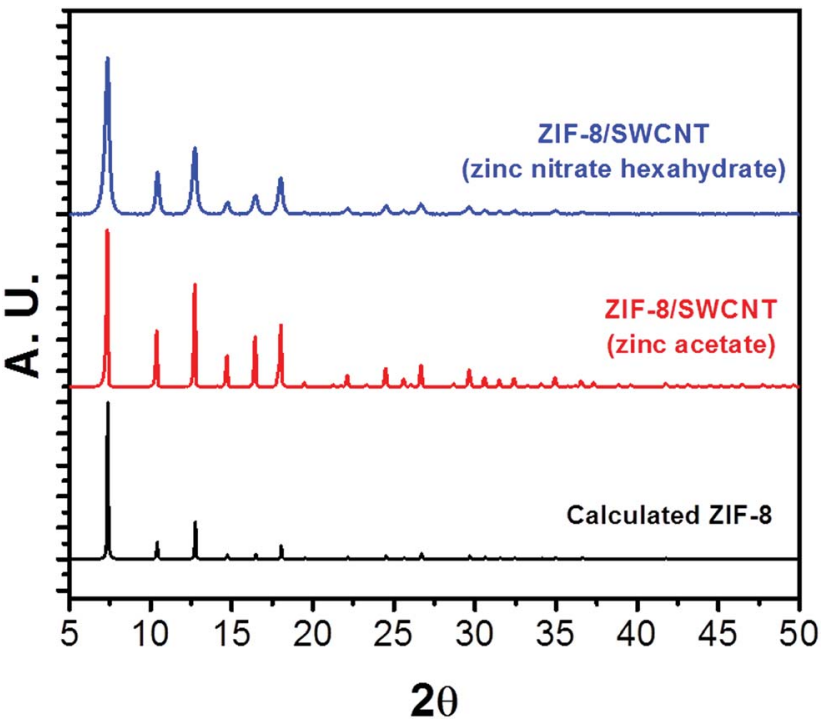

Fig. 2 Calculated PXRD pattern of ZIF-8 (bottom); experimental PXRD pattern of ZIF-8/SWCNT synthesized from zinc acetate (middle) and zinc nitrate hexahydrate (top).

clearly show crystallites that are coalescing into larger crystals (Fig. 1c and d). This observation is consistent with the fast nucleation rate of zinc nitrate with $2 \mathrm{mIM}$ to form clusters, which are more likely to form small crystallites compared to zinc acetate. ${ }^{37} \mathrm{~A}$ time-dependent experiment was done on material 3 (ZIF-8/SWCNT synthesized from zinc nitrate hexahydrate) to observe the growth mechanism of ZIF-8 on SWCNTs (Fig. S7†). Within 15 minutes, most SWCNTs are covered with small crystals around $50 \mathrm{~nm}$ in diameter (Fig. S7, $\dagger 15 \mathrm{~min}$ ). At 30 minutes, the crystals have grown 10 -fold in size and smaller crystal domains seem to be agglomerating into large crystals (Fig. S7, $\dagger 30 \mathrm{~min}$ ). At the 2 hour and 20 hour time points, the ZIF-8 crystals have reached their characteristic size $(1-1.5 \mu \mathrm{m})$, but remain in a linear configuration along SWCNTs (Fig. S7, $\uparrow$ $2 \mathrm{~h}$ and $20 \mathrm{~h}$ ).

The zinc: $2 \mathrm{mIM}$ ratio controls what type of polymorph/ pseudopolymorph is produced in water-based syntheses. ZIF-L and $\operatorname{dia}(\mathrm{Zn})$ are polymorphs comprised of $\mathrm{Zn}(\mathrm{II})$ and $2 \mathrm{mIM}$, but with different crystal topologies than ZIF-8. ${ }^{36,38,39}$ Control of $\mathrm{Zn}$ (II) : $2 \mathrm{mIM}$ ratio and temperature allows one polymorph to be favored over the other two during aqueous synthesis. The crystal morphology of ZIF-L resembles a thin, almost two-dimensional leaf-like shape, while ZIF-8's crystal morphology is rhombic dodecahedral. Unlike ZIF-8, ZIF-L and dia(Zn) are nonporous at $77 \mathrm{~K}$; however, at $298 \mathrm{~K}$, ZIF-L outperforms ZIF-8 in $\mathrm{CO}_{2}$ uptake and selectivity over $\mathrm{N}_{2}$ and $\mathrm{CH}_{4} \cdot{ }^{38}$ High ratios $(1: 35$ and above) produce ZIF-8, while lower ratios produce ZIF-L, dia(Zn), or a mixture of one with ZIF-8. A low zinc : 2 mIM ratio (1:8) using zinc acetate produces pure dia $(\mathrm{Zn})$ while zinc nitrate produces pure ZIF-L. ${ }^{9}$ A low ratio synthesis was performed with 2mIMsaturated ox-SWCNTs (material 4). XRD of this material yields a mixture of ZIF-8 and ZIF-L peak features (Fig. S8 $\dagger$ ); moreover, TEM shows large ( $>3 \mu \mathrm{m})$ crystals (Fig. S9†) with a morphology unlike ZIF-8 (rhombic dodecahedron) or ZIF-L (thin, leaf- 
shaped) (Fig. S14B $\dagger$ ). This product can be interpreted as the result of two different reaction environments, solution and ox-SWCNT surface, which contain different precursor ratios. $2 \mathrm{mIM}$ is expected to concentrate at the surface of ox-SWCNTs through $\pi$-stacking, which will promote ZIF-8 crystal growth. In contrast, the solution zinc: $2 \mathrm{mIM}$ ratio remains $1: 8$, leading to ZIF-L crystal growth. It has been previously reported that when separately synthesized ZIF-8 and ZIF-L crystals are combined into a single solution, they will coalesce into a new core-shell composite. ${ }^{40}$ The addition of ox-SWCNTs into low zinc: $2 \mathrm{mIM}$ ratio solutions produces similar ZIF-L/ZIF-8/ ox-SWCNT composites in a one-pot synthesis. When the 1:8 $\mathrm{Zn}: 2 \mathrm{mIM}$ synthesis was done with zinc acetate as precursor (material 5), the morphology was pure dia(Zn). Zinc acetate has a slower rate of cluster nucleation, which may explain why only a single morphology was observed for material $5 .^{37}$

Decreasing the overall precursor concentration slows the kinetics of the reaction and achieves smaller ZIF-8 coverage on the surface of ox-SWCNTs. $10 \mathrm{mM}$ and $6.6 \mathrm{mM}$ zinc acetate reactions (1:40 zinc:2mIM ratio) were done with 2mIMsaturated ox-SWCNTs and collected after 24 hours (materials 6 and 7 , respectively). Both syntheses produced a smaller ZIF-8 crystal shell growth (approximately $90 \mathrm{~nm}$ and $50 \mathrm{~nm}$, respectively) on the ox-SWCNTs (Fig. S10 and S11†). However, the decrease in precursor concentration also lowers the $\mathrm{pH}$, such that pseudopolymorph dia(Zn) (Fig. S14A $\dagger$ ) forms in solution if the reaction is left for more than 24 hours (Fig. S12, S13, and S15 $\dagger$ ).

The gas adsorption performance of ZIF-8/SWCNT (shown in Fig. 1a and b) was tested (Fig. 3). ZIF-8/SWCNT displayed a typeI isotherm and the BET surface area of ZIF-8/SWCNT was calculated to be $1792 \mathrm{~m}^{2} \mathrm{~g}^{-1}$, which is near the reported BET surface area of pure ZIF-8 $\left(1851 \mathrm{~m}^{2} \mathrm{~g}^{-1}\right) .{ }^{41}$ The $\mathrm{CO}_{2}$ vs. $\mathrm{N}_{2}$ uptake at $298 \mathrm{~K}$ was tested and showed the same preference for $\mathrm{CO}_{2}$ uptake as pure ZIF-8 (Fig. S16†), indicating that these composite materials may exhibit selectivity for specific gases. In order to test $I-V$ performance, a $3 \mu \mathrm{L}$ droplet of ZIF-8/SWCNT solution

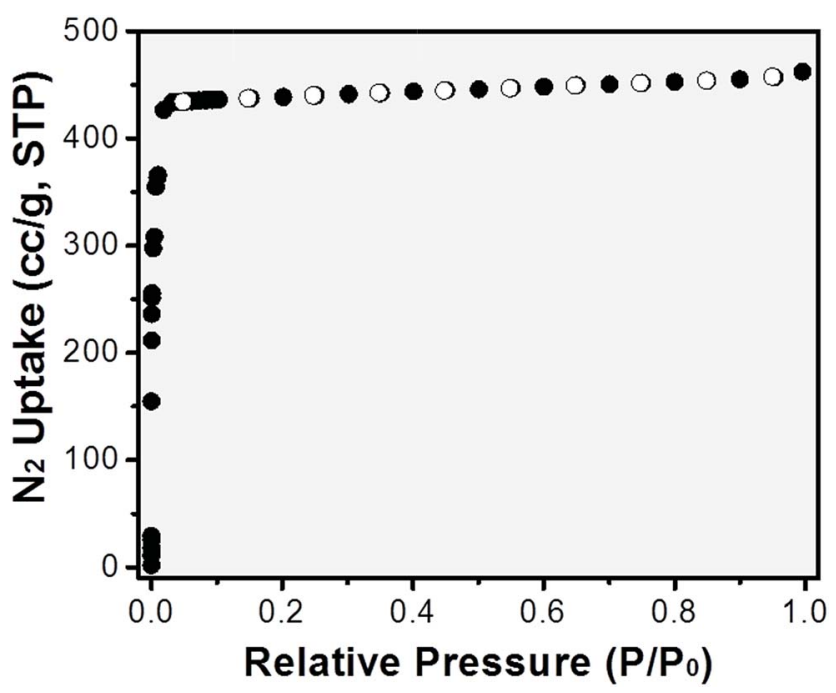

Fig. $3 \mathrm{~N}_{2}$ adsorption (black) and desorption (white) isotherm of ZIF-8/ SWCNT at $77 \mathrm{~K}$. was dropcast on a device of two interdigitated gold electrodes (Fig. S17†). The ZIF-8/SWCNT current-voltage curve displays a conductivity of $0.056 \mathrm{~S} \mathrm{~cm}^{-1}$ (Fig. 4a). Conductivity $(\sigma)$ was calculated from the following equation:

$$
\sigma=\frac{1}{\rho \times \frac{A}{L}}
$$

where $\rho$ is resistance $\left(3.9 \times 10^{4} \Omega\right), A$ is area of the electrode $\left(3.25 \times 10^{-7} \mathrm{~cm}^{2}\right)$, and $L$ is length of the electrode $\left(7 \times 10^{-4} \mathrm{~cm}\right)$.

This conductivity is on the higher end for conductive 3-D MOFs, which range from $1 \times 10^{-7}$ to $1.8 \mathrm{~S} \mathrm{~cm}^{-1}$. $^{42,43}$ Among the conductive MOF/carbon composites, this work demonstrated the highest BET surface area and achieved conductivity with the least amount of carbon loading $(0.40 \pm 0.09 \%$; see Table S3 $\dagger) .^{\mathbf{4 4 , 4 5}}$ Carbon nanotube loading was calculated by dividing the initial amount of carbon nanotube by the total mass of the ZIF-8/SWCNT product, filtered and dried. These experiments demonstrate both the highly microporous and electrically conductive nature of the reported ZIF-8/SWCNT composite.

Liquid FET measurements of ZIF-8/SWCNT (material 2) demonstrate p-type semiconductor behavior (Fig. 4b). Semiconducting nanowires are ideal for gas sensing because of their large surface area to volume ratio, their sensitivity toward doping interactions, and their sensitivity toward changes in internanowire distance. ${ }^{46}$ Both the SWCNT and ZIF-8/SWCNT devices showed a decrease in conductance when exposed to 3 cycles of air saturated with ethanol vapor (7.73 vol\%) (Fig. $4 \mathrm{c}$ and $\mathrm{d}$ ). The sensitivity to ethanol vapor was quantified by calculating the relative sensor response $(S)$ :

$$
S=\frac{I_{\text {ethanol }}-I_{\text {air }}}{I_{\text {air }}}
$$
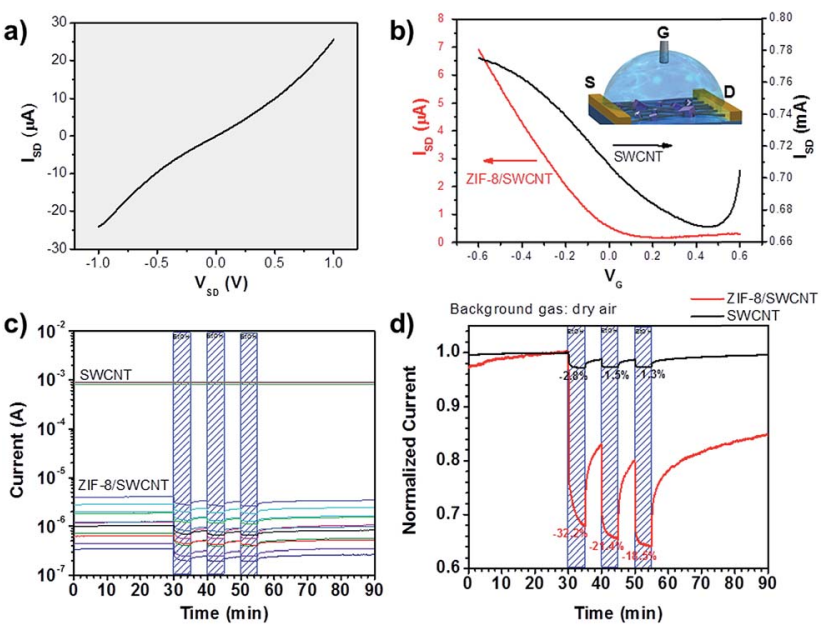

Fig. 4 ZIF-8/SWCNT (material 2) electrical characterization and gas sensing. (a) Current-voltage curve of ZIF-8/SWCNT composite. (b) Liquid FET of ZIF-8/SWCNT and bare SWCNT; inset, illustration of liquid FET. (c) Current traces of SWCNT and ZIF-8/SWCNT chemiresistors exposed to three 5 minute cycles of saturated ethanol vapor (blue rectangles). Background gas is dry air. (d) Normalized (at $t=$ $30 \mathrm{~min}$ ) current traces of representative SWCNT and ZIF-8/SWCNT chemiresistor devices. 
which is the relative change in the current before $\left(I_{\text {air }}\right)$ and after 5 minutes of ethanol exposure $\left(I_{\text {ethanol }}\right)$. The ZIF-8/SWCNT devices had relative response values ranging from $-18.5 \%$ to $-32.2 \%$, compared to $-1.3 \%$ to $-2.8 \%$ for bare SWCNT devices (Fig. 4d). A possible explanation for the increase in sensitivity to ethanol vapor after ZIF-8 functionalization is due to the ZIF-8 adsorbing and swelling in volume when ethanol binds to its pores. Adsorption of alcohols, including ethanol, on ZIF crystals features an S-shaped isotherms, which is ascribed to a "cluster formation and cage-filling" mechanism. ${ }^{47,48}$ The filling of ZIF-8 pores with volatile organic compound (VOC) vapors has been utilized in a Fabry-Pérot optical sensor. ${ }^{49}$ We hypothesize that as the ZIF-8 swells upon ethanol adsorption, the interface distance between nanotubes can increase, which results in an increase of the electron tunneling distance between the nanotubes. ${ }^{\mathbf{5 0}-52}$ The larger, more irreversible response of the ZIF-8/SWCNT devices also supports the ZIF-8 adsorption and swelling mechanism. A more detailed follow up study will be conducted to further elucidate the sensing mechanism for MOF functionalized CNT sensors.

\section{Conclusions}

In conclusion, this work demonstrates the molecular ordering of 2-methylimidazole (2mIM) as a solvation layer on the surface of oxidized single-walled carbon nanotubes (ox-SWCNTs). This 2mIM solvation layer gives rise to ZIF-8 growth on ox-SWCNT sidewalls. The high concentration of $2 \mathrm{mIM}$ on ox-SWCNTs produces ZIF-8 at the surface of the nanotube even at low zinc : 2mIM ratios that typically produce ZIF-L. The size of ZIF-8 growth on ox-SWCNTs can be controlled by precursor concentration, wherein decreasing the precursor concentration decreases the size of ZIF-8. The ZIF-8/SWCNT composite was shown to combine the porous nature of ZIF-8 with the electrically conductive nature of carbon nanotubes. Finally, the ZIF-8/ SWCNT material was incorporated into a chemiresistor device and demonstrated a higher sensitivity toward saturated ethanol vapor versus pristine SWCNT. Such a highly porous, electrically conductive nanomaterial will be of interest for a variety of applications such as sensing, electrocatalysis, and energy storage.

\section{Conflicts of interest}

There are no conflicts to declare.

\section{Acknowledgements}

Raman characterization was completed through the assistance of a ONR (N000141410765) grant. We thank the Department of Biological Sciences and the Nanoscale Fabrication and Characterization Facility (NFCF) at the University of Pittsburgh for access to the TEM, SEM, and XRD instrumentation. We especially thank Dr Susheng Tan for the help he provided in collecting HRTEM images of material 6. JJG and SL acknowledge UTD for provided start-up funds.

\section{References}

1 L. Sun, M. G. Campbell and M. Dinca, Angew. Chem., Int. Ed., 2016, 55, 3566-3579.

2 L. Mu, B. Liu, H. Liu, Y. Yang, C. Sun and G. Chen, J. Mater. Chem., 2012, 22, 12246.

3 K. Liang, C. J. Coghlan, S. G. Bell, C. Doonan and P. Falcaro, Chem. Commun., 2016, 52, 473-476.

4 H. He, H. Han, H. Shi, Y. Tian, F. Sun, Y. Song, Q. Li and G. Zhu, ACS Appl. Mater. Interfaces, 2016, 8, 24517-24524.

5 N. K. Maddigan, A. Tarzia, D. M. Huang, C. J. Sumby, S. G. Bell, P. Falcaro and C. J. Doonan, Chem. Sci., 2018, 9, 4217-4223.

6 K. S. Park, Z. Ni, A. P. Cote, J. Y. Choi, R. Huang, F. J. UribeRomo, H. K. Chae, M. O'Keeffe and O. M. Yaghi, Proc. Natl. Acad. Sci. U. S. A., 2006, 103, 10186-10191.

7 R. Banerjee, A. Phan, B. Wang, C. Knobler, H. Furukawa, M. O'Keeffe and O. M. Yaghi, Science, 2008, 319, 939-943.

8 Y. Pan, Y. Liu, G. Zeng, L. Zhao and Z. Lai, Chem. Commun., 2011, 47, 2071-2073.

9 M. Jian, B. Liu, R. Liu, J. Qu, H. Wang and X. Zhang, RSC Adv., 2015, 5, 48433-48441.

10 S. J. Yang, J. Y. Choi, H. K. Chae, J. H. Cho, K. S. Nahm and C. R. Park, Chem. Mater., 2009, 21, 1893-1897.

11 Z. Xiang, X. Peng, X. Cheng, X. Li and D. Cao, J. Phys. Chem. C, 2011, 115, 19864-19871.

12 J. Yoo, S. Lee, C. K. Lee, C. Kim, T. Fujigaya, H. J. Park, N. Nakashima and J. K. Shim, RSC Adv., 2014, 4, 49614-49619.

13 S. Sohrabi, S. Dehghanpour and M. Ghalkhani, J. Mater. Sci., 2017, 53, 3624-3639.

14 Q. Wang, Q. Wang, B. Xu, F. Gao, F. Gao and C. Zhao, Electrochim. Acta, 2018, 281, 69-77.

15 H. Zhang, W. Zhao, M. Zou, Y. Wang, Y. Chen, L. Xu, H. Wu and A. Cao, Adv. Energy Mater., 2018, 8, 1800013.

16 Y. Pu, W. Wu, J. Liu, T. Liu, F. Ding, J. Zhang and Z. Tang, RSC Adv., 2018, 8, 18604-18612.

17 L. Ge, Y. Yang, L. Wang, W. Zhou, R. De Marco, Z. Chen, J. Zou and Z. Zhu, Carbon, 2015, 82, 417-424.

18 Y. Pan, K. Sun, S. Liu, X. Cao, K. Wu, W. C. Cheong, Z. Chen, Y. Wang, Y. Li, Y. Liu, D. Wang, Q. Peng, C. Chen and Y. Li, J. Am. Chem. Soc., 2018, 140, 2610-2618.

19 H. Zhang, Y. Wang, W. Zhao, M. Zou, Y. Chen, L. Yang, L. Xu, $\mathrm{H}$. $\mathrm{Wu}$ and A. Cao, ACS Appl. Mater. Interfaces, 2017, 9, 37813-37822.

20 K. P. Prasanth, P. Rallapalli, M. C. Raj, H. C. Bajaj and R. V. Jasra, Int. J. Hydrogen Energy, 2011, 36, 7594-7601.

21 S. N. Kim, J. F. Rusling and F. Papadimitrakopoulos, Adv. Mater., 2007, 19, 3214-3228.

22 H. R. Byon and H. C. Choi, J. Am. Chem. Soc., 2006, 128, 2188-2189.

23 S. Boussaad, B. A. Diner and J. Fan, J. Am. Chem. Soc., 2008, 130, 3780-3787.

24 J. E. Ellis, U. Green, D. C. Sorescu, Y. Zhao and A. Star, J. Phys. Chem. Lett., 2015, 6, 712-717.

25 G. Peng, S. Wu, J. E. Ellis, X. Xu, G. Xu, C. Yu and A. Star, J. Mater. Chem. C, 2016, 4, 6575-6580. 
26 S. Ishihara, C. J. O'Kelly, T. Tanaka, H. Kataura, J. Labuta, Y. Shingaya, T. Nakayama, T. Ohsawa, T. Nakanishi and T. M. Swager, ACS Appl. Mater. Interfaces, 2017, 9, 38062-38067. 27 C. Paoletti, M. He, P. Salvo, B. Melai, N. Calisi, M. Mannini, B. Cortigiani, F. G. Bellagambi, T. M. Swager, F. Di Francesco and A. Pucci, RSC Adv., 2018, 8, 5578-5585.

28 Z. Ghiamaty, A. Ghaffarinejad, M. Faryadras, A. Abdolmaleki and H. Kazemi, J. Nanostruct. Chem., 2016, 6, 299-308.

29 S. Li, M. Dharmarwardana, R. P. Welch, Y. Ren, C. M. Thompson, R. A. Smaldone and J. J. Gassensmith, Angew. Chem., Int. Ed., 2016, 55, 10691-10696.

30 S. Li, M. Dharmarwardana, R. P. Welch, C. E. Benjamin, A. M. Shamir, S. O. Nielsen and J. J. Gassensmith, ACS Appl. Mater. Interfaces, 2018, 10, 18161-18169.

31 Y. Shim and H. J. Kim, ACS Nano, 2009, 3, 1693-1702.

32 U. D. Venkateswaran, A. M. Rao, E. Richter, M. Menon, A. Rinzler, R. E. Smalley and P. C. Eklund, Phys. Rev. B, 1999, 59, 10928-10934.

33 S. B. Cronin, A. K. Swan, M. S. Ünlü, B. B. Goldberg, M. S. Dresselhaus and M. Tinkham, Phys. Rev. B, 2005, 72.

34 R. Voggu, C. S. Rout, A. D. Franklin, T. S. Fisher and C. N. R. Rao, J. Phys. Chem. C, 2008, 112, 13053-13056.

35 T. Fukushima, A. Kosaka, Y. Ishimura, T. Yamamoto, T. Takigawa, N. Ishii and T. Aida, Science, 2003, 300, 2072-2074.

36 Y. Lo, C. H. Lam, C.-W. Chang, A.-C. Yang and D.-Y. Kang, RSC Adv., 2016, 6, 89148-89156.

37 S. Tanaka, T. Shimada, K. Fujita, Y. Miyake, K. Kida, K. Yogo, J. F. M. Denayer, M. Sugita and T. Takewaki, J. Membr. Sci., 2014, 472, 29-38.

38 R. Chen, J. Yao, Q. Gu, S. Smeets, C. Baerlocher, H. Gu, D. Zhu, W. Morris, O. M. Yaghi and H. Wang, Chem. Commun., 2013, 49, 9500-9502.
39 Q. Shi, Z. Chen, Z. Song, J. Li and J. Dong, Angew. Chem., Int. Ed., 2011, 50, 672-675.

40 W. C. Lee, H. T. Chien, Y. Lo, H. C. Chiu, T. P. Wang and D. Y. Kang, ACS Appl. Mater. Interfaces, 2015, 7, 18353-18361.

41 F. M. Hinterholzinger, A. Ranft, J. M. Feckl, B. Rühle, T. Bein and B. V. Lotsch, J. Mater. Chem., 2012, 22, 10356.

42 S. K. Bhardwaj, N. Bhardwaj, R. Kaur, J. Mehta, A. L. Sharma, K.-H. Kim and A. Deep, J. Mater. Chem. A, 2018, 6, 1499215009.

43 L. S. Xie, L. Sun, R. Wan, S. S. Park, J. A. DeGayner, C. H. Hendon and M. Dinca, J. Am. Chem. Soc., 2018, 140, 7411-7414.

44 O. Fleker, A. Borenstein, R. Lavi, L. Benisvy, S. Ruthstein and D. Aurbach, Langmuir, 2016, 32, 4935-4944.

45 D. Kim, D. W. Kim, W. G. Hong and A. Coskun, J. Mater. Chem. A, 2016, 4, 7710-7717.

46 J. F. Fennell Jr, S. F. Liu, J. M. Azzarelli, J. G. Weis, S. Rochat, K. A. Mirica, J. B. Ravnsbaek and T. M. Swager, Angew. Chem., Int. Ed., 2016, 55, 1266-1281.

47 K. Zhang, R. P. Lively, M. E. Dose, A. J. Brown, C. Zhang, J. Chung, S. Nair, W. J. Koros and R. R. Chance, Chem. Commun., 2013, 49, 3245-3247.

48 A. Nalaparaju, X. S. Zhao and J. W. Jiang, J. Phys. Chem. C, 2010, 114, 11542-11550.

49 G. Lu and J. T. Hupp, J. Am. Chem. Soc., 2010, 132, 78327833.

50 C. Li, E. T. Thostenson and T.-W. Chou, Appl. Phys. Lett., 2007, 91, 223114.

51 H. C. Wang, Y. Li and M. J. Yang, Sens. Actuators, B, 2007, 124, 360-367.

52 S. F. Liu, L. C. H. Moh and T. M. Swager, Chem. Mater., 2015, 27, 3560-3563. 